\title{
A speed-gradient-based method to passify nonlinear discrete-time systems
}

Link to publication record in Manchester Research Explorer

\section{Citation for published version (APA):}

Navarro-López, E. M. (2005). A speed-gradient-based method to passify nonlinear discrete-time systems. In IFAC Proceedings Volumes (IFAC-PapersOnline)/IFAC Proc. Vol. (IFAC-PapersOnline) (Vol. 16, pp. 300-305). Elsevier BV.

\section{Published in:}

IFAC Proceedings Volumes (IFAC-PapersOnline)|IFAC Proc. Vol. (IFAC-PapersOnline)

\section{Citing this paper}

Please note that where the full-text provided on Manchester Research Explorer is the Author Accepted Manuscript or Proof version this may differ from the final Published version. If citing, it is advised that you check and use the publisher's definitive version.

\section{General rights}

Copyright and moral rights for the publications made accessible in the Research Explorer are retained by the authors and/or other copyright owners and it is a condition of accessing publications that users recognise and abide by the legal requirements associated with these rights.

\section{Takedown policy}

If you believe that this document breaches copyright please refer to the University of Manchester's Takedown Procedures [http://man.ac.uk/04Y6Bo] or contact uml.scholarlycommunications@manchester.ac.uk providing relevant details, so we can investigate your claim.

\section{OPEN ACCESS}




\title{
A SPEED-GRADIENT-BASED METHOD TO PASSIFY NONLINEAR DISCRETE-TIME SYSTEMS *
}

\author{
E.M. Navarro-López \\ Programa de Investigación en Matemáticas Aplicadas y \\ Computación, Instituto Mexicano del Petróleo \\ Eje Central Lázaro Cárdenas, 152, A.P. 14-805, 07730 Mexico \\ navarro.lopez@gmail.com
}

\begin{abstract}
This paper proposes a method in order to render passive nonlinear affine-incontrol discrete-time systems. The methodology is based on the discrete-time version of the speed-gradient (SG) algorithm. For the application of the SG algorithm, quasi$V$-passive and feedback quasi- $V$-passive systems are introduced. Two kinds of feedback laws rendering the system locally quasi- $V$-passive are obtained: a dynamic one and a static one. The passification methodology is applied to two examples. Some frequency-domain properties of the feedback transformed system are highlighted for the linear example Copyright $@ 2005$ IFAC
\end{abstract}

Keywords: Discrete-time systems, Nonlinear systems, Energy control, Passive elements, Feedback stabilization.

\section{INTRODUCTION}

Passive systems present highly desirable properties which may simplify the system analysis and control design (Hill and Moylan, 1980). This fact impels to transform a system which is not passive into a passive one. The action of rendering a system passive by means of a state feedback is known as feedback passivity. Systems which can be rendered passive are regarded as feedback passive systems. Several results concerning feedback passivity and feedback dissipativity in the nonlinear discrete-time setting are reported in the literature. Necessary and sufficient conditions have been proposed in (Byrnes and Lin, 1994) and (Navarro-López and Fossas-Colet, 2002) for the feedback losslessness and feedback passivity cases, respectively, for multiple-input multiple-output (MIMO) affine-in-input nonlinear discrete-time systems with the restriction of considering storage func-

\footnotetext{
* This paper has been done in the context of IMP project D.00334 "Optimización de la Toma de Decisiones en Escenarios 3I" and LAFMAA project under CONACYT grant.
}

tions $V$ such that $V(f(x)+g(x) u)$ is quadratic in $u$. In these works, the feedback passivity problem is based on the properties of the relative degree and zero dynamics of the non-passive system and the approach is inherited from the continuous-time case (Byrnes et al., 1991). These results have been recently extended in (Navarro-López and Fossas-Colet, 2004) to general systems without requiring $V(f(x, u))$ to be quadratic in $u$. The feedback dissipativity property is treated for single-input single-output (SISO) nonlinear discrete-time systems of general form in (NavarroLópez, 2002; Navarro-López et al., 2002).

The problem of feedback passivity for continuoustime nonlinear systems is solved by means of the SG algorithm, for example, in (Fradkov, 1991; Fradkov et al., 1995). The discrete-time version of the SG methodology is given in (Fradkov and Pogromsky, 1998).

In the present paper, the feedback passivity problem is considered for MIMO nonlinear discrete-time systems which are affine in the input. A class of passive systems regarded as quasi- $V$-passive systems is used. 
A method for aforementioned systems to be rendered locally feedback quasi- $V$-passive is proposed and use is made of the SG algorithm in its discrete-time version. Two kinds of solutions are obtained, and they are illustrated in two examples.

The paper is organized as follows. Section 2 gives the basic definitions to use in the sequel. Section 3 presents the discrete-time version of the SG algorithm which will be adapted so as to achieve local feedback quasi-passivity of nonlinear discrete-time systems. In Sections 4 and 5 , the feedback passivity methodology is illustrated by means of two examples: a discretetime model of the DC-to-DC buck converter and an academic nonlinear discrete-time system. The validity of the passifying controls given will be analyzed for each example. The motivation of including a linear example (the buck converter) is to interpret the results in the frequency domain, and the interest in including the nonlinear example is illustrating the technique for a more general example. For the linear example, an interpretation of the passified system properties in the frequency domain is given in Section 6. Conclusions are presented in the last section.

\section{BASIC DEFINITIONS}

Let a system of the form,

$$
\begin{aligned}
x(k+1) & =f(x(k))+g(x(k)) u(k), \\
y(k) & =h(x(k))+J(x(k)) u(k),
\end{aligned}
$$

where $f(x), g(x), h(x), J(x)$ are smooth maps and $f(x) \in \mathscr{X} \subset \mathfrak{R}^{n}, g(x) \in \mathscr{G} \subset \mathfrak{R}^{n \times m}, h(x) \in \mathscr{Y} \subset \mathfrak{R}^{m}$, $J(x) \in \mathscr{J} \subset \mathfrak{R}^{m \times m}, x \in \mathscr{X}, u \in \mathscr{U} \subset \mathfrak{R}^{m}, k \in \mathscr{Z}_{+}=$ $\{0,1,2, \cdots\}$. All considerations are restricted to an open set of $\mathscr{X} \times \mathscr{U}$ containing $(\bar{x}, \bar{u})$, having $\bar{x}$ as an isolated fixed point of $f(x)+g(x) \bar{u}$ with $\bar{u}$ constant, i.e., $f(\bar{x})+g(\bar{x}) \bar{u}=\bar{x}$.

A positive definite $\mathscr{C}^{2}$ function $V: \mathscr{X} \rightarrow \mathfrak{R}$ such that $V(0)=0 \Longleftrightarrow x=0$ is addressed as storage function. Another $\mathscr{C}^{2}$ function denoted by $s(y, u)$ with $s: \mathscr{Y} \times$ $\mathscr{U} \rightarrow \mathfrak{R}$ is addressed as supply function. For the passivity case $s(y, u)=y^{T} u$. A $\mathscr{C}^{2}$ function $\phi: \mathscr{X} \times$ $\mathscr{U} \rightarrow \mathfrak{R}$, such that $\phi(\cdot, u)$ is positive for each $u \in \mathscr{U}$, with $\phi(0,0)=0$, is regarded as a dissipation rate function in the sense proposed in (Hill and Moylan, 1980; Navarro-López et al., 2002).

The application of the SG method gives rise to two types of feedback control laws. Let $\alpha: \mathscr{X} \times \mathscr{U} \rightarrow \mathscr{U}$ be a smooth function. A static state feedback control law $u=\alpha(x, v)$ is regular if for all $(x, v) \in \mathscr{X} \times \mathscr{U}$ it follows that $\frac{\partial \alpha}{\partial v}$ is invertible. Let $\beta: \mathscr{X} \times \mathscr{U} \times$ $\mathscr{U} \rightarrow \mathscr{U}$ be a smooth function. A dynamic feedback control law $u(k+1)=\beta(x(k), u(k), v(k))$ is regular if $\frac{\partial \beta}{\partial v}$ is invertible for all $(x, u, v) \in \mathscr{X} \times \mathscr{U} \times$ $\mathscr{U}$. System (1) with $u=\alpha(x(k), v(k))$ or $u(k+1)=$ $\beta(x(k), u(k), v(k))$ is referred as the feedback transformed system.
In order to use the SG algorithm for passifying purposes, the following definitions are introduced, which are based on the passivity definiton in the nonlinear discrete-time setting given in (Byrnes and Lin, 1994; Navarro-López et al., 2002).

Definition 1. System (1) with storage function $V(x)$ and supply function $s(y, u)=(h(x)+J(x) u)^{T} u$ is said to be locally quasi- $V$-passive if there exists a dissipation rate function $\phi$ and a constant $\Delta \geq 0$ such that,

$$
\begin{aligned}
& V(f(x)+g(x) u)-V(x)-s(y, u)+ \\
& +\phi(x, u) \leq \Delta, \quad \forall(x, u) \in \mathscr{X} \times \mathscr{U}
\end{aligned}
$$

Definition 2. Consider system (1). Assume that there exists a storage function $V(x)$ and consider a supply function $s=y^{T} v$. The system is said to be locally feedback quasi- $V$-passive if there exists a regular static state feedback control law of the form $u(k)=$ $\alpha(x(k), v(k))$ or a regular dynamic feedback control law of the form $u(k+1)=\beta(x(k), u(k), v(k))$, with $v$ as the new input, such that the feedback transformed system is locally quasi- $V$-passive.

\section{THE SG ALGORITHM AND THE LOCAL FEEDBACK PASSIVITY PROBLEM}

The SG method, see (Fradkov, 1991), is based upon the achievement of a specified control goal by means of some goal function $Q$. The discrete-time version of the SG algorithm is proposed in (Fradkov and Pogromsky, 1998).

Consider system (1). The SG method is formulated as finding a control law $u(k)$ which ensures the control objective

$$
Q(x(k+1)) \leq \Delta, \text { when } k>k^{*}
$$

with some nonnegative function $Q$ and a threshold value $\Delta>0$. Substituting (1a) into (3), the following goal function is obtained,

$$
Q_{k}(u)=Q\left(f_{k}(x(k), u)\right)
$$

The following control algorithm is proposed (Fradkov and Pogromsky, 1998):

$$
u(k+1)=u(k)-\gamma(k) \nabla_{u} Q_{k}(u(k))
$$

where $\gamma(k) \geq 0, \forall k$. The partial derivative of $Q$ with respect to $u$ is denoted by $\nabla_{u} Q_{k}(u(k))$.

The SG method in the discrete-time setting and its applicability conditions are based upon the following theorem:

Theorem 3. (Fradkov and Pogromsky, 1998) Consider control algorithm (5). Let $Q$ be a nonnegative function and $\Delta$ a positive constant. Suppose that:

A1 There exists $\varepsilon^{*}>0$ and a vector $u^{*}$ such that the following inequalities are satisfied:

$$
Q_{k}\left(u^{*}\right) \leq \varepsilon^{*}<\Delta, k=0,1,2, \ldots
$$


A2 For all admissible inputs $u$, the inequality $Q_{k}(u) \leq$ $\Delta$ implies the fullfilment of one of the following conditions,

$$
\begin{aligned}
& \left(u^{*}-u\right)^{T} \nabla_{u} Q_{k}(u) \leq \varepsilon^{*}-\Delta<0, \\
& \left(u^{*}-u\right)^{T} \nabla_{u} Q_{k}(u) \leq Q_{k}\left(u^{*}\right)-Q_{k}(u)<0
\end{aligned}
$$

A3 For any $\rho>0$ and any $k=0,1,2, \ldots$ there exists $\kappa(\rho)>0$ such that the following inequalities are satisfied,

$$
\left|\nabla_{u} Q_{k}(u)\right|^{2} \leq \kappa(\rho)
$$

as long as $\left|u-u^{*}\right| \leq \rho$ and $Q_{k}(u)>\Delta$.

A4 The gain coefficients $\gamma(k)$ in the proposed control (5) are chosen as follows

$$
\gamma(k)=\gamma_{c} \delta(k)\left|\nabla_{u} Q_{k}(u(k))\right|^{-2}
$$

where (if condition (7) is satisfied)

$0<\gamma_{c}<2\left(\Delta-\varepsilon^{*}\right), \delta(k)= \begin{cases}1, & \text { if } Q_{k}(u(k)) \geq \Delta, \\ 0, & \text { otherwise }\end{cases}$

or (if condition (8) is satisfied)

$$
\begin{aligned}
& 0<\gamma_{c}<2\left(1-\frac{\varepsilon^{*}}{\Delta}\right), \\
& \delta(k)= \begin{cases}Q_{k}(u(k)), & \text { if } Q_{k}(u(k)) \geq \Delta, \\
0, & \text { otherwise }\end{cases}
\end{aligned}
$$

Then, for any $u_{0}$ there exists a number $k^{*}$ such that the goal inequality (3) is fulfilled and $u(k)$ is constant for $k \geq k^{*}$.

In (Fradkov and Pogromsky, 1998), it is established that the SG algorithm (5) can be rewritten as

$$
u(k)=-\gamma_{c s} \nabla_{u(k)} Q(x(k))
$$

i.e., $u(k)$ is explicitly obtained from $\nabla_{u} Q_{k}(u(k))$, and $\gamma_{c s}$ is a positive constant. $\gamma_{c s}$ is chosen in such a way to assure the asymptotic stability of the fixed point of the controlled system obtained with (11).

Now, the discrete-time version of the SG algorithm is adapted to propose a method in order to render systems of the form (1) locally feedback quasi- $V$ passive. For this purpose, the following control goal is considered,

$$
Q_{d}(x, u, v)=V(f(x)+g(x) u)-V(x)-y^{T} v+\phi(x, u)
$$

Proposition 4. Let a system of the form (1). Consider $V(x)$ and $s=(h(x)+J(x) u)^{T} v$ as the storage function and the supply rate function, respectively. Consider a control goal function $Q_{d}$ as defined in (12) with the dissipation rate function $\phi$ such that makes $Q_{d}$ be nonnegative. Let $\Delta$ and $\varepsilon^{*}$ be positive constants such that $\Delta$ and $\varepsilon^{*}$ are close to zero, and $u_{*} \in \mathscr{U}$ is a constant vector. Assume that assumptions A1, A3 and condition (8) of Theorem 3 are satisfied, then the system is locally feedback quasi- $V$-passive by means of two control laws, such as:
(1) A dynamic feedback:

$$
\begin{aligned}
u(k+1) & =u(k)-\gamma(k) \nabla_{u} Q_{d, k}(u(k)) \\
\gamma(k) & =\gamma_{c} \delta(k)\left|\nabla_{u} Q_{d, k}(u(k))\right|^{-2} \\
0 & <\gamma_{c}<2 \\
\delta(k) & =\left\{\begin{array}{l}
Q_{d, k}(u(k)), \text { if } Q_{d, k}(u(k)) \geq \Delta \\
0, \text { otherwise }
\end{array}\right.
\end{aligned}
$$

(2) A static feedback:

$$
u(k)=-\gamma_{c s} \nabla_{u(k)} Q_{d}(x(k))
$$

with $\gamma_{c s}$ a positive constant ensuring the asymptotic stability of the fixed point of the controlled system.

Proof The proof follows the same arguments as the ones given for Theorem 3 in (Fradkov and Pogromsky, 1998) with $\varepsilon^{*}<<\Delta$. It is concluded that there exists $k^{*}$ for which $Q_{d, k}(u(k)) \leq \Delta$ for all $k \geq k^{*}$. Considering $Q_{d}$ as defined in (12), inequality (2) is satisfied and, consequently, the feedback transformed system with (13) is locally quasi- $V$-passive. As it is established in (Fradkov and Pogromsky, 1998), control (13) can be written as (14).

The passifying methodology shown, in its two versions, will be applied to passify two examples in the next two sections. An analysis of the constants appearing in the controls will be made. The conditions under which the SG algorithm can be applied will be also verified. Although the feedback passivity methodology is applicable to MIMO systems, the examples are SISO for the sake of simplicity in computations.

\section{LINEAR EXAMPLE. THE DISCRETIZED BUCK CONVERTER MODEL}

Consider a discrete-time model of a normalized averaged DC-to-DC buck converter derived in (NavarroLópez et al., 2002):

$$
\begin{aligned}
x(k+1) & =A x(k)+B u(k), \\
y(k) & =C x(k)+D u(k)
\end{aligned}
$$

with,

$$
\begin{array}{ll}
A=\left(\begin{array}{cc}
a & -b \\
b & c
\end{array}\right) & B=\left(\begin{array}{c}
(-a+1) \gamma_{b}+b \\
-b \gamma_{b}-c+1
\end{array}\right) \\
C=(0,1) & D=
\end{array}
$$

where $u \in[0,1], x=\left(x_{1}, x_{2}\right)^{T}, x_{1}$ is a normalized current, $x_{2}$ a normalized voltage, $\gamma_{b}$ the normalized load, and $a, b, c$, constants related to physical parameters, with $a=0.9406416964, b=0.3254699438$, $c=0.8255706942, \gamma_{b}=0.353553, \eta=13.25192$, obtained for a sampling period of $T=0.3535533906$.

The system is not passive and the goal is to render it locally quasi- $V$-passive having as storage function 
$V=\frac{\eta}{2}\left(x_{1}^{2}+x_{2}^{2}\right)$ and as supply function $s=y^{T} v$, with $y=x_{2}+u$. Function $\phi(x, u)$ is chosen in order to collect the positive terms of $V(x(k+1))$, and takes the following form:

$$
\begin{aligned}
\phi(x, u)= & \eta \mu\left\{x_{1}^{2}\left(a^{2}+b^{2}\right)+x_{2}^{2}\left(b^{2}+c^{2}\right)+u^{2}\left[\gamma_{b}^{2}(1-\right.\right. \\
& \left.\left.-a)^{2}+b^{2}\left(1+\gamma_{b}^{2}\right)+(-c+1)^{2}\right]\right\}
\end{aligned}
$$

with $\mu$ a positive constant. Let $x_{1} \in\left[0, \gamma_{b} \rho\right], x_{2} \in[0, \rho]$, $u, v \in[0,1]$, and $\rho>1$.

The control goal function for the example is:

$$
Q_{d}\left(x_{1}, x_{2}, u, v\right)=a_{u} u^{2}+b_{u}\left(x_{1}, x_{2}, v\right) u+c_{u}\left(x_{1}, x_{2}, v\right),
$$

where

$$
\begin{aligned}
a_{u}= & \eta\left[( \frac { 1 } { 2 } + \mu ) \left[\gamma_{b}^{2}(1-a)^{2}+b^{2}\left(\gamma_{b}^{2}+1\right)+\right.\right. \\
& \left.\left.+(-c+1)^{2}\right]+\gamma_{b}(b c-a b)\right] \\
b_{u}\left(x_{1}, x_{2}, v\right)= & \eta\left\{\left[\gamma_{b}(-a+1)+b\right]\left(a x_{1}-b x_{2}\right)+\right. \\
& \left.+\left(-\gamma_{b} b-c+1\right)\left(b x_{1}+c x_{2}\right)\right\}-v \\
c_{u}\left(x_{1}, x_{2}, v\right)= & \eta\left\{( \frac { 1 } { 2 } + \mu ) \left[x_{1}^{2}\left(a^{2}+b^{2}\right)+x_{2}^{2}\left(b^{2}+\right.\right.\right. \\
& \left.\left.\left.+c^{2}\right)\right]+(b c-a b) x_{1} x_{2}-\frac{1}{2}\left(x_{1}^{2}+x_{2}^{2}\right)\right\}- \\
& -x_{2} v
\end{aligned}
$$

For the application of the SG algorithm for feedback passivity purposes, it is necessary to assure that the function $Q_{d}$ is positive, which will be achieved by means of choosing the adequate value of the parameter $\mu$. In addition, conditions of Theorem 3 must be checked.

First, let examine the positiveness of $Q_{d}$. A way of assuring the positiveness of $Q_{d}$ is by means of assuring that $Q_{d}$ is a positive function for all the candidates for local minima. The candidates for local minima are the critical points of $Q_{d}$ and the local minima existing in the domain of definition of $Q_{d}\left(x_{1}, x_{2}, u, v\right)$, i.e., $\left[0, \gamma_{b} \rho\right] \times[0, \rho] \times[0,1] \times[0,1]$. Examining $Q_{d}$, it can be noticed that $Q_{d}$ is positive for a value of $\mu$ and ahead, then, the worst case to search is the greatest value of $\mu$, among all the cases examined, for which $Q_{d}$ becomes positive. The first candidate to be a relative minimum of $Q_{d}$ is the critical point $\bar{x}=\left(x_{1}, x_{2}, u, v\right)^{T}=(0,0,0, \bar{v})^{T}$, with $\bar{v}$ a constant. A sufficient condition for this is that the Hessian matrix of $Q_{d}(H)$ at $\bar{x}$ is positive definite. The real symmetric matrix $H$ is positive definite if all eigenvalues are positive, which is achieved for $\mu>\bar{\mu}_{1}=0.296853$. Furthermore, the study of the positiveness of $Q_{d}$ is necessary for other local minima candidates. By analyzing the domain of function $Q_{d}$, a lower bound of $\mu\left(\bar{\mu}_{2}\right)$ can be found for which $Q_{d}$ is positive at all the candidates for local minima. The worst case is presented for $x=(0, \rho, 1,1)$ with $\rho=1$, and the critical value of $\mu$ is given by $\bar{\mu}_{2}=0.276418$. The value of $\mu$ is chosen as $\mu>\max \left(\bar{\mu}_{1}, \bar{\mu}_{2}\right)$. It is concluded that $Q_{d}$ is positive for $\mu>\bar{\mu}_{1}=0.296853$ (see Figure 1).

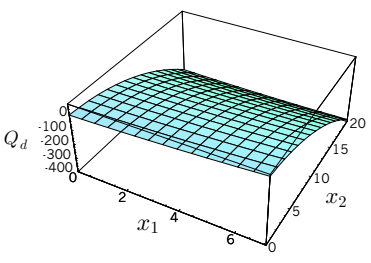

(i)

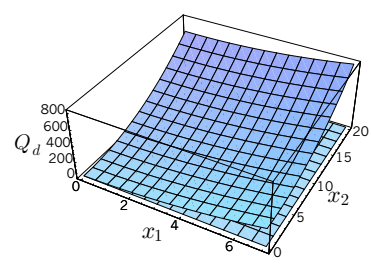

(ii)
Fig. 1. $Q_{d}$ is positive depending on the value of $\mu$. Representation of $Q_{d}$ with $x_{1} \in\left[0, \gamma_{b} \rho\right], x_{2} \in$ $[0, \rho], \rho=20$ : (i) with $u=1, v=0.5, \mu=0.05$; (ii) with $u=1, v=0.5, \mu=0.3$.

Second, assumptions A1, A3 and condition (8) must be verified. From the fact that for $\mu>0.296853$ it follows that $Q_{d}>0$ and $Q_{d}$ has a relative minimum at $x=0, u=0$ with $v=\bar{v}$ a constant, and due to the increasing nature of $Q_{d}$ in the domain considered, the conditions appearing in $\mathrm{A} 1$ and (8) are met. On the one hand, the condition to verify in A1 is met due to the fact that $Q_{d}$ is positive with a relative minimum at $(x=$ $0, u=0, \bar{v})$, then a constant $0<\varepsilon^{*}<\Delta$ can be always found and a control $u^{*}$ for which $Q_{d}$ is smaller and then the control goal (3) is achieved, indeed, this $u^{*}$ can be $u^{*}=0$ considering $x_{1}, x_{2}, v$ fixed near to zero. On the other hand, condition (8) is met considering $u^{*}=0$. This condition takes the following form:

$$
-\left(2 a_{u} u^{2}+b_{u} u\right) \leq-\left(a_{u} u^{2}+b_{u} u\right)<0
$$

which is always met. For the condition of assumption A3 that $\nabla_{u} Q(u)=2 a_{u} u+b_{u}$ must be bounded, the property of uniform continuity in a set can be considered (Marsden and Hoffman, 1998). Then, controls (13), (14) can be applied and two passifying control schemes are obtained:

(1) From (13), a dynamic passifying control:

$$
\begin{aligned}
& u(k+1)=u(k)-\gamma(k)\left[2 a_{u} u(k)+\right. \\
& \left.+b_{u}\left(x_{1}(k), x_{2}(k), v(k)\right)\right] \\
& \gamma(k)=\gamma_{c} \delta(k)\left|2 a_{u} u(k)+b_{u}\left(x_{1}(k), x_{2}(k), v(k)\right)\right|^{-2} \\
& 0<\gamma_{c}<2 \\
& \delta(k)=\left\{\begin{array}{l}
Q_{d, k}(u(k)), \text { if } Q_{d, k}(u(k)) \geq \Delta, \\
0, \text { otherwise }
\end{array}\right.
\end{aligned}
$$

(2) From (14), a static passifying control:

$$
u(k)=-\frac{\gamma_{c s} b_{u}\left(x_{1}(k), x_{2}(k), v(k)\right)}{1+2 \gamma_{c s} a_{u}}
$$

with $\gamma_{c s}$ a positive constant ensuring the fixed point of the controlled system to be asymptoti- 
cally stable. If control (20) is applied to (15), a linear system is obtained:

$$
\begin{aligned}
x(k+1) & =\bar{A} x(k)+\bar{B} v(k) \\
y(k) & =\bar{C} x(k)+\bar{D} v(k)
\end{aligned}
$$

with matrices $\bar{A}, \bar{B}, \bar{C}, \bar{D}$ depending on the system parameters and the constants, $\mu, \eta, \gamma_{c s}$. Matrix $\bar{A}$ of the quasi- $V$-passified system (21) depends on the constants $\mu$ and $\gamma_{c s}$. These constants will be chosen in order to have the eigenvalues of $\bar{A}$ with modulus less than 1 , and it is concluded that $\mu>0.296853$ and $\gamma_{c s}>0$.

\section{A NONLINEAR EXAMPLE}

Let consider a nonlinear discrete-time system extracted from (Sira-Ramírez, 1991):

$$
\begin{aligned}
x_{1}(k+1) & =\left[x_{1}^{2}(k)+x_{2}^{2}(k)+u(k)\right] \cos \left[x_{2}(k)\right] \\
x_{2}(k+1) & =\left[x_{1}^{2}(k)+x_{2}^{2}(k)+u(k)\right] \sin \left[x_{2}(k)\right] \\
y(k) & =x_{1}^{2}(k)+x_{2}^{2}(k)+u(k)
\end{aligned}
$$

The goal is to render system (22) locally quasi- $V$ passive with a storage function $V=x_{1}^{2}+x_{2}^{2}$ and with a supply function $s(y, v)=y v$. Function $\phi(x, u)$ is chosen in order to collect the positive terms of $V(x(k+1))$ :

$$
\phi(x, u)=\mu\left[\left(x_{1}^{2}+x_{2}^{2}\right)^{2}+u^{2}+x_{1}^{2}+x_{2}^{2}\right]
$$

with $\mu>0$. Suppose $x_{1}, x_{2} \in\left[-\rho_{x}, \rho_{x}\right], u \in\left[-\rho_{u}, \rho_{u}\right]$, $v \in\left[-\rho_{v}, \rho_{v}\right]$, with $\rho_{x}, \rho_{u}, \rho_{v}$ positive constants. The control goal function takes the following form,

$$
\begin{array}{r}
Q_{d}\left(x_{1}, x_{2}, u, v\right)=u^{2}(1+\mu)+2 u\left(x_{1}^{2}+x_{2}^{2}\right)+\left(x_{1}^{2}+x_{2}^{2}\right) \\
{\left[(1+\mu)\left(x_{1}^{2}+x_{2}^{2}\right)+\mu-1\right]-y v}
\end{array}
$$

Function $Q_{d}$ will be assured to be positive in all candidates for local minima in the domain of definition of $Q_{d}$ by means of the value of $\mu$. Two groups of local minima have to be analyzed: the critical points of $Q_{d}$ and other candidates for minima in the domain $\left[-\rho_{x}, \rho_{x}\right] \times\left[-\rho_{x}, \rho_{x}\right] \times\left[-\rho_{u}, \rho_{u}\right] \times\left[-\rho_{v}, \rho_{v}\right]$. Following the same procedure as the one given for the linear example, it is concluded that $Q_{d}$ becomes positive for $\mu>1$. In addition, with this $\mu$, the critical point $\left(x_{1}, x_{2}, u, v\right)=(0,0,0, \bar{v})$, with $\bar{v}$ a constant, is assured to be a relative minimum of $Q_{d}$ (see Figure 2).

Second, the conditions of Proposition 4 are verified. From the fact that for $\mu>1$, it follows that $Q_{d}$ is a positive function with a relative minimum at $(x=$ $0, u=0, v=\bar{v})$, the condition appearing in $\mathrm{A} 1$ is met, for $u^{*}=0$ and $x_{1}, x_{2}, v$ fixed near to zero and condition (8) is met. Condition (8) takes the form:

$$
\begin{aligned}
& -\left[2 u^{2}(1+\mu)+2 u\left(x_{1}^{2}+x_{2}^{2}\right)-u v\right] \leq \\
& -\left[u^{2}(1+\mu)+2 u\left(x_{1}^{2}+x_{2}^{2}\right)-u v\right]<0
\end{aligned}
$$

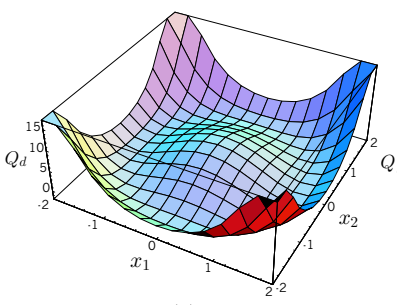

(i)

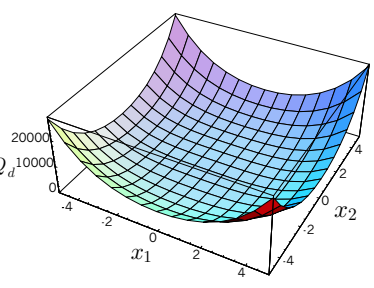

(ii)
Fig. 2. Representation of $Q_{d}$ : (i) with $x_{1}, x_{2} \in[-2,2]$, $v=0, \mu=0.06, u=-2$; (ii) with $x_{1}, x_{2} \in[-5,5]$, $v=0, \mu=10, u=2$.

which is always met. For the condition of assumption A3 that $\nabla_{u} Q_{d}(u)=2 u(1+\mu)+2\left(x_{1}^{2}+x_{2}^{2}\right)-v$ must be bounded, the property of uniform continuity in a set is applied (Marsden and Hoffman, 1998). Therefore, two passifying controls are obtained:

(1) A dynamic feedback:

$$
\begin{aligned}
u(k+1)= & u(k)-\gamma(k)\{2 u(k)(1+\mu)+ \\
& \left.+2\left[x_{1}^{2}(k)+x_{2}^{2}(k)\right]-v(k)\right\} \\
\gamma(k)= & \gamma_{c} \delta(k) \mid 2 u(k)(1+\mu)+ \\
& +2\left[x_{1}^{2}(k)+x_{2}^{2}(k)\right]-\left.v(k)\right|^{-2} \\
\delta(k)= & \left\{\begin{array}{l}
Q_{d, k}(u(k)), \text { if } Q_{d, k}(u(k)) \geq \Delta, \\
0, \text { otherwise }
\end{array}\right.
\end{aligned}
$$

with $\mu>1$ and $0<\gamma_{c}<2$.

(2) A static feedback:

$$
u(k)=-\frac{\gamma_{c s}\left[2 x_{1}^{2}(k)+2 x_{2}^{2}(k)-v(k)\right]}{1+2 \gamma_{c s}(1+\mu)}
$$

with $\mu>1$ and $\gamma_{c s}>0$ ensuring the asymptotic stability of the fixed point of the controlled system. Applying control (27) to system (22), the following locally quasi- $(V, s)$-passive system is obtained:

$$
\begin{aligned}
& x(k+1)=\bar{f}[x(k)]+\bar{g}[x(k)] v(k) \\
& y(k)=\bar{h}[x(k)]+\bar{J}[x(k)] v(k)
\end{aligned}
$$

with $x=\left(x_{1}, x_{2}\right)^{T}$. A way to ensure the local asymptotic stability of the fixed point of system (28) is by assuring that the linearized system around the fixed point has poles with modulus less than 1 , and this is assured for any $\mu>1$ and $\gamma_{c s}>0$.

\section{A NOTE ON THE FREQUENCY-DOMAIN CHARACTERISTICS OF QUASI- $V$-PASSIVE SYSTEMS}

Passive systems exhibit special features in the frequency domain. Passivity, for linear systems, is equivalent to positive realness of a transfer function. The same is for the discrete-time case. Positive realness of 
a transfer function can be identified via the Nyquist diagram which is confined in the right-hand side half of the Nyquist plane. In addition, discrete positive real transfer functions do not have poles with modulus greater than one, and their poles lying on $|z|=1$ are simple with positive real residues.

The example of the buck passified by means of the SG-based feedback passivity method can be used for giving a first step in analyzing the implications of quasi- $V$-passive systems in the frequency domain. The example can illustrate the properties of the Nyquist of a quasi- $V$-passive system. The Nyquist plot of the non-passive original system can be compared with the quasi- $V$-passive system obtained after the feedback passivity control scheme (20) is applied. The pulse transfer function for the state-space description (15) with $y=x_{2}+u$ is:

$$
G_{b}(z)=\frac{z^{2}-1.707 z+0.9394}{z^{2}-1.766 z+0.8825}
$$

and for the passified system (21):

$$
G_{b}(z)=\frac{0.07794\left(z^{2}-1.766 z+0.8825\right)}{z^{2}-1.763 z+0.8789}
$$

The Nyquist plots of (29) and (30) are depicted in Figure 3. The Nyquist of the quasi- $V$-passive system lies on the right-hand side half plane, but it does not touch the axis $\operatorname{Re}\left[G\left(e^{j \omega}\right)\right]=0$. The Nyquist plot begins at $\operatorname{Re}\left[G\left(e^{j \omega}\right)\right]=\Delta$.
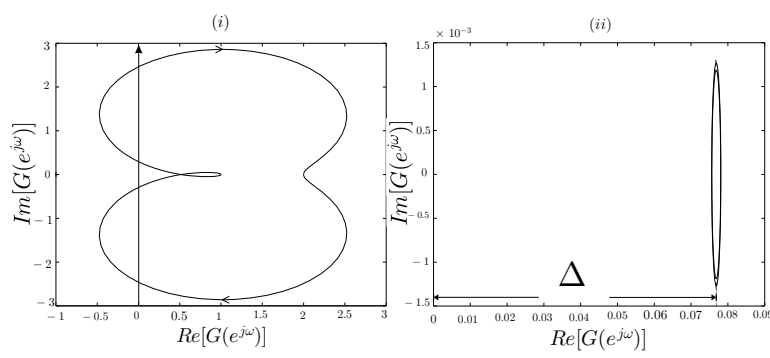

Fig. 3. (i) Nyquist diagram for the exact discretized model of the buck converter with $y=x_{2}+u$; (ii) Nyquist diagram for the passified buck converter by means of the SG-based feedback passivity method.

\section{CONCLUSIONS}

The discrete-time version of the SG algorithm has been used in order to render passive MIMO nonlinear discrete-time systems which are affine in the input. A subclass of passive systems have been introduced regarded as quasi- $V$-passive systems. Two kinds of passifying feedback laws have been obtained derived from the application of the SG method: a dynamic one and a static one. The feedback passivity methodology proposed is based on the establishment of the input $u$ which satisfies the basic passivity equality, which is interpreted as a goal function. Two SISO examples have illustrated the methodology. Some comments on the frequency-domain implications of linear quasi- $V$ passive systems have also been pointed out.

An alternative way of designing the parameters appearing in the passifying controls may be given, as well as, a more detailed study of the influence of these controller parameters in the system response.

\section{REFERENCES}

Byrnes, C.I., A. Isidori and J.C. Willems (1991). Passivity, feedback equivalence, and the global stabilization of minimum phase nonlinear systems. IEEE Transactions on Automatic Control 36, 1228-1240.

Byrnes, C.I. and W. Lin (1994). Losslessness, feedback equivalence, and the global stabilization of discrete-time nonlinear systems. IEEE Transactions on Automatic and Control 39(1), 83-98.

Fradkov, A.L. (1991). Speed-gradient laws of control and evolution. European Control Conference pp. 1861-1865.

Fradkov, A.L. and A.Y. Pogromsky (1998). Introduction to Control, Oscillations and Chaos. World Scientific Publishing Co.

Fradkov, A.L., D.J. Hill, Z-P. Jiang and M.M. Seron (1995). Feedback passification of interconnected systems. IFAC NOLCOS 2, 660-665.

Hill, D.J. and P. Moylan (1980). Dissipative dynamical systems: basic input-output and state properties. Journal of the Franklin Institute 309, 327-357.

Marsden, J.E. and M.J. Hoffman (1998). Análisis Clásico Elemental. Addison-Wesley Iberoamericana, 2nd ed.

Navarro-

López, E.M. (2002). Dissipativity and passivityrelated properties in nonlinear discrete-time systems. Ph.D. thesis. Universitat Politècnica de Catalunya, Barcelona, Spain. ISBN: 84688-1941-7. In http://www.tdx.cesca.es/TDCat0328103-104942/.

Navarro-López, E.M. and E. Fossas-Colet (2002). Dissipativity, passivity and feedback passivity in the nonlinear discrete-time setting. Proceedings of the 15th IFAC World Congress.

Navarro-López, E.M. and E. Fossas-Colet (2004). Feedback passivity of nonlinear discrete-time systems with direct input-output link. Automatica 40(8), 1423-1428.

Navarro-López, E.M., H. Sira-Ramírez and E. FossasColet (2002). Dissipativity and feedback dissipativity properties of general nonlinear discretetime systems. European Journal of Control 8(3), 265-274.

Sira-Ramírez, H. (1991). Non-linear discrete variable structure systems in quasi-sliding mode. International Journal of Control 54(5), 1171-1187. 